\title{
Mapping observations of complex organic molecules around Sagittarius B2 with the ARO $12 \mathrm{~m}$ telescope
}

\author{
Juan Li ${ }^{\oplus}, 1,2 \star$ Junzhi Wang, ${ }^{1,2 \star ~ H a i h u a ~ Q i a o, ~}{ }^{1,2,3}$ Donghui Quan, ${ }^{4,5}$ Min Fang, ${ }^{6}$ \\ Fujun Du, ${ }^{2,7}$ Fei Li, ${ }^{1,2,8}$ Zhiqiang Shen, ${ }^{1,2}$ Shanghuo Li, ${ }^{1,2,8} \mathrm{Di} \mathrm{Li}^{\oplus}, 9,10$ \\ Yong Shi ${ }^{\oplus}{ }^{11,12,13}$ Zhiyu Zhang ${ }^{11,12}$ and Jiangshui Zhang ${ }^{14}$ \\ ${ }^{1}$ Department of Radio Science and Technology, Shanghai Astronomical Observatory, 80 Nandan Rd, Shanghai 200030, China \\ ${ }^{2}$ Key Laboratory of Radio Astronomy, Chinese Academy of Sciences, 2 West Beijing Road, Nanjing, Jiangsu 210008, China \\ ${ }^{3}$ National Time Service Center, Chinese Academy of Sciences, Xi'An, Shaanxi 710600, PR China \\ ${ }^{4}$ Xinjiang Astronomical Observatory, Chinese Academy of Sciences, 150 Science 1-Street, Urumqi 830011, PR China \\ ${ }^{5}$ Department of Chemistry, Eastern Kentucky University, Richmond, KY 40475, USA \\ ${ }^{6}$ Department of Astronomy, University of Arizona, 933 North Cherry Avenue, Tucson, AZ 85721, USA \\ ${ }^{7}$ Purple Mountain Observatory, Chinese Academy of Sciences, Nanjing 210034, PR China \\ ${ }^{8}$ University of Chinese Academy of Sciences, 19A Yuquanlu, Beijing 100049, PR China \\ ${ }^{9}$ CAS Key Laboratory of FAST, National Astronomical Observatories, Chinese Academy of Sciences, Beijing 100101, China \\ ${ }^{10}$ University of Chinese Academy of Sciences, Beijing 100049, PR China \\ ${ }^{11}$ School of Astronomy and Space Science, Nanjing University, Nanjing 210093, China \\ ${ }^{12}$ Key Laboratory of Modern Astronomy and Astrophysics (Nanjing University), Ministry of Education, Nanjing 210093, China \\ ${ }^{13}$ Collaborative Innovation Center of Modern Astronomy and Space Exploration, Nanjing 210093, China \\ ${ }^{14}$ Center for Astrophysics, Guangzhou University, Guangzhou 510006, PR China
}

\begin{abstract}
We have performed high-sensitivity mapping observations of several complex organic molecules around Sagittarius B2 with the ARO $12 \mathrm{~m}$ telescope at $3 \mathrm{~mm}$ wavelength. Based on their spatial distribution, molecules can be classified as either 'extended', those detected not only in Sgr B2(N) and Sgr B2(M), or 'compact', those only detected toward or near Sgr B2(N) and Sgr B2(M). The 'extended' molecules include glycolaldehyde $\left(\mathrm{CH}_{2} \mathrm{OHCHO}\right)$, methyl formate $\left(\mathrm{CH}_{3} \mathrm{OCHO}\right)$, formic acid $(\mathrm{t}-\mathrm{HCOOH})$, ethanol $\left(\mathrm{C}_{2} \mathrm{H}_{5} \mathrm{OH}\right)$ and methyl amine $\left(\mathrm{CH}_{3} \mathrm{NH}_{2}\right)$, while the 'compact' molecules include dimethyl ether $\left(\mathrm{CH}_{3} \mathrm{OCH}_{3}\right)$, ethyl cyanide $\left(\mathrm{C}_{2} \mathrm{H}_{5} \mathrm{CN}\right)$, and amino acetonitrile $\left(\mathrm{H}_{2} \mathrm{NCH}_{2} \mathrm{CN}\right)$. These 'compact' molecules are likely produced under strong UV radiation, while the 'extended' molecules are likely formed at low temperatures, via gas-phase or grain-surface reactions. The spatial distribution of 'warm' $\mathrm{CH}_{2} \mathrm{OHCHO}$ at $89 \mathrm{GHz}$ differs from the spatial distribution of 'cold' $\mathrm{CH}_{2} \mathrm{OHCHO}$ observed at $13 \mathrm{GHz}$. We found evidence for an overabundance of $\mathrm{CH}_{2} \mathrm{OHCHO}$ compared to that expected from the gas-phase model, which indicates that grain-surface reactions are necessary to explain the origin of $\mathrm{CH}_{2} \mathrm{OHCHO}$ in Sagittarius B2. Grain-surface reactions are also needed to explain the correlation between the abundances of 'cold' $\mathrm{CH}_{2} \mathrm{OHCHO}$ and $\mathrm{C}_{2} \mathrm{H}_{5} \mathrm{OH}$. These results demonstrate the importance of grain-surface chemistry in the production of complex organic molecules.
\end{abstract}

Key words: ISM: abundances - ISM: clouds-ISM: individual (Saggitarius B2)-ISM: molecules - radio lines: ISM.

\section{INTRODUCTION}

Complex organic molecules (COMs) are regarded as being related to the origin of life on Earth (Herbst \& van Dishoeck 2009). Thus,

\footnotetext{
^E-mail: lijuan@shao.ac.cn (JL); jzwang@ @shao.ac.cn (JW)
}

understanding the properties and formation mechanism of prebiotic molecules is key for the study of astrobiology. It has been found that prebiotic molecules are very abundant in the Galactic centre giant molecular cloud Sagittarius B2 (Sgr B2) (Menten 2011). Sgr B2 contains two main sites of star formation, Sgr B2(N) and Sgr B2(M), which have been the best hunting ground for prebiotic molecules in the interstellar medium (ISM) since the early 1970s (Belloche 
et al. 2013). Many COMs are first detected toward Sgr B2(N), such as glycoaldehyde $\mathrm{CH}_{2} \mathrm{OHCHO}$ (Hollis, Lovas \& Jewell 2000), ethylene glycol $\mathrm{HOCH}_{2} \mathrm{CH}_{2} \mathrm{OH}$ (Hollis, Lovas \& Jewell 2002), amino acetonitrile $\mathrm{H}_{2} \mathrm{NCH}_{2} \mathrm{CN}$ (Belloche et al. 2008) and so on. Some COMs, such as glycolaldehyde and ethylene glycol, were found to be widespread in the Galactic centre (Hollis et al. 2001; Requena-Torres et al. 2006, 2008; Li et al. 2017).

Theoretical and experimental studies (Garrod, Weaver \& Herbst 2008; Öberg et al. 2009; Woods et al. 2013; Fedoseev et al. 2015; Chuang et al. 2016; Meinert et al. 2016; Skouteris et al. 2018) show that COMs may be formed on cosmic dust by radicals that are produced on dust grain surfaces by UV or cosmic ray induced photons. The COMs are then desorbed into the gas phase due to thermal and/or non-thermal processes (Garrod \& Herbst 2006). However, these complex ice compositions cannot be directly observed with telescopes in the infrared; thus, most constraints come from millimetre observations of desorbed ice chemistry products. On the other hand, recent quantum chemical computations show that a combination of radicals trapped in amorphous water ice does not necessarily lead to the formation of COMs (EnriqueRomero et al. 2016). Recently, a new scheme for the gas-phase synthesis of glycolaldehyde, a species with prebiotic potential, has been proposed. The predicted abundance of glycolaldehyde agrees well with that measured in solar-type hot corinos and shock sites (Skouteris et al. 2018). Thus, how these species are synthesized is a mystery.

Previous mapping observations mainly concentrated on Sgr B2(N) with interferometers like ATCA and ALMA at small spatial scales ( $\leq 1$ arcmin) (Corby et al. 2015; Belloche et al. 2016, 2019; Sánchez-Monge et al. 2017; Bonfand et al. 2019; Xue et al. 2019), and only a handful of large-scale spectral line mapping observations toward Sgr B2 have been performed (Chengalur \& Kanekar 2003; Jones et al. 2008, 2011). The large-scale spatial distribution of COMs like $\mathrm{C}_{2} \mathrm{H}_{5} \mathrm{CN}$, $\mathrm{HCOOH}$ is still unclear mainly because of their weak emission (Halfen, Woolf \& Ziurys 2017). Single-dish telescopes have the ability to probe the structure of weak lines from the extended gas of the Sgr B2 complex on spatial scales much larger than 1 arcmin. In this paper we present high-sensitivity gridimaging results of several COMs around Sgr B2 with the Arizona Radio Observatory (ARO) $12 \mathrm{~m}$ telescope. In Section 2, we describe the observations and data reduction. In Section 3, we present the mapping results and spatial distributions of column densities, rotational temperatures, and estimated abundances for the observed COMs. In Section 4, we discuss molecular formation pathways and compare our results with protostars and then summarize our conclusions in Section 5.

\section{OBSERVATIONS AND DATA REDUCTION}

We performed point-by-point mapping observations of COMs at a $3 \mathrm{~mm}$ wavelength around Sgr B2 in 2017 April and May with the Arizona Radio Observatory $12 \mathrm{~m}$ telescope at Kitt Peak, Arizona. The 85-116 GHz sideband separating ALMA band-3 receiver was used. The beam size is 70 arcsec at $90 \mathrm{GHz}$. The two IF modes of the Millimeter Autocorrelator (MAC) were employed as backends. The MAC has 4096 channels with $195 \mathrm{kHz}$ sampling interval, corresponding to $0.65 \mathrm{~km} \mathrm{~s}^{-1}$ at $90 \mathrm{GHz}$. Though the velocity resolution is not high enough for the study of molecular emission from hot cores, it is sufficient for single-dish observations of Sgr B2. The FWHM linewidth is up to $\sim 20 \mathrm{~km} \mathrm{~s}^{-1}$ (Rivilla et al. 2018, 2019) due to the extended envelope and several unresolved cores with different velocities and linewidths within the beam. The temperature scale was determined by the chopper wheel method, and is related to the main-beam brightness temperature with a main-beam efficiency of 0.88 . The system temperature ranges from $90-150 \mathrm{~K}$. The integration time for each position ranges from 50-90 $\mathrm{min}$.

Observations were made in position-switching mode, and an offset of +30 arcmin in azimuth was used. The data processing was conducted using the GILDAS software package, ${ }^{1}$ including CLASS and GREG. Linear baseline subtractions were used for most of the spectra. For each transition, the spectra of subscans, including two polarizations, were averaged to reduce rms noise levels. Gaussian fitting is used to derive the physical properties of molecule lines, including peak intensity, $V_{\mathrm{LSR}}$, FWHM linewidth, and integrated intensity.

\section{RESULTS OF OBSERVATIONS}

We detected emission lines from t- $\mathrm{HCOOH}, \mathrm{C}_{2} \mathrm{H}_{5} \mathrm{CN}, \mathrm{CH}_{3} \mathrm{OCH}_{3}$, $\mathrm{CH}_{3} \mathrm{OCHO}, \mathrm{H}_{2} \mathrm{NCH}_{2} \mathrm{~N}, \mathrm{CH}_{2} \mathrm{OHCHO}, \mathrm{CH}_{3} \mathrm{NH}_{2}$, and $\mathrm{C}_{2} \mathrm{H}_{5} \mathrm{OH}$ toward many positions at a rms level of $\sim 3 \mathrm{mK}$. Fig. 1 presents spectra observed toward several positions with the ARO $12 \mathrm{~m}$ telescope. As is shown in Fig. 1, some molecule lines are only detected toward Sgr B2(N) and Sgr B2(M). Table 1 presents detected transitions and their spectroscopic properties in Sgr B2(N). The molecular spectroscopic parameters are taken from the current public databases: the CDMS catalogue ${ }^{2}$ (Müller et al. 2005), the JPL catalogue ${ }^{3}$ (Pickett et al. 1998) and the Spectral Line Atlas of Interstellar Molecules (SLAIM) database, which are available in the SPLATALOGUE spectroscopy database. ${ }^{4}$ Note that the strong $\mathrm{C}_{2} \mathrm{H}_{5} \mathrm{CN}$ emission near to t-HCOOH 4(0,4)-3(0,3) $(89.57917 \mathrm{GHz})$ makes it difficult to determine the baseline and intensity of t$\mathrm{HCOOH}$. Only t-HCOOH emission toward Sgr B2(N) was affected since the $\mathrm{C}_{2} \mathrm{H}_{5} \mathrm{CN}$ emission was very weak toward other positions.

The many hazards of searching for new molecules have been pointed out by Snyder et al. (2005). These authors proposed some of the criteria needed for secure molecular identification, including accurate rest frequencies and consistency among the acquired data set. Halfen et al. (2006) extended the criteria in Snyder et al. (2005) and applied them to the ARO $12 \mathrm{~m}$ observations. They suggest that, for an accurate identification, there must be strong evidence for emission at all favourable physically connected transitions over a sufficiently large wavelength range. There cannot be 'missing' favourable lines. Based on these criteria, $\mathrm{CH}_{2} \mathrm{OHCHO} 9(1,8)-$ $8(2,7)(89.868639 \mathrm{GHz})$, the glycolaldehyde transition used in this work, was found to be strong and relatively clean. Xue et al. (2019) proposed a quantitative method to identify weak and mostly uncontaminated transitions. They used the $\mathrm{P}$ factor, which is the product of the observed and simulated line profiles, to characterize the frequency agreement. They used the D factor, which is the ratio between the difference of the integrated intensities of the compared spectra and the maximum of the two subtracted from 1, to measure the difference of the line intensities. For $\mathrm{CH}_{2} \mathrm{OHCHO}$ $9(1,8)-8(2,7)$ at $89.868639 \mathrm{GHz}$, the $\mathrm{P}$ factor is 94 percent, while the D factor is 16.8 percent. For $\mathrm{CH}_{3} \mathrm{OCHO} 7(2,5)-6(2,4) \mathrm{E}$ at $90.145 \mathrm{GHz}$, the $\mathrm{P}$ factor is 99.8 percent, while the $\mathrm{D}$ factor is 25.4 percent (see the appendix in Xue et al. 2019). Based on the $\mathrm{P}$ factors, both of these transitions are assigned to be unblended

\footnotetext{
${ }^{1}$ http: / / www . iram. fr/IRAMFR/GILDAS

${ }^{2}$ https: / / www .astro.uni-koeln.de/cdms/catalog

${ }^{3}$ https://spec.jpl.nasa.gov

${ }^{4}$ https: / / www. splatalogue. net
} 

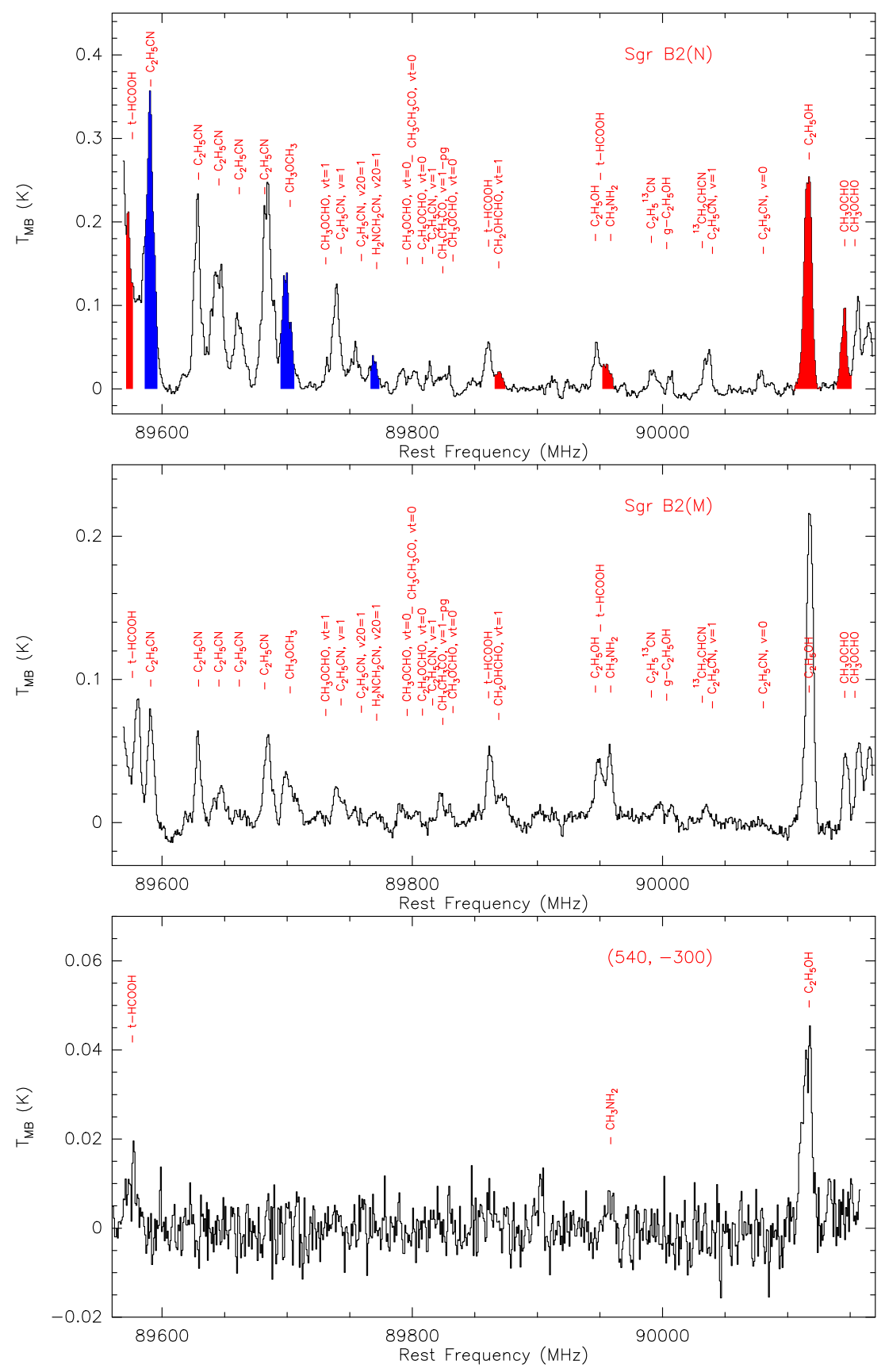

Figure 1. Spectra obtained toward Sgr B2(N), Sgr B2(M) and $(540,-300)$ away from Sgr B2(N) with the ARO $12 \mathrm{~m}$ telescope in the main-beam temperature scale. The line names are shown. The 'extended' molecular line profiles are coloured red, while the 'compact' molecular line profiles are coloured blue in the spectra of Sgr B2(N).

transition candidates. The low $\mathrm{D}$ factors are likely to be caused by the high excitation temperature (190 K) adopted by Xue et al. (2019). Transitions of other molecules have been identified by Belloche et al. (2013). Velocity-integrated images of strong and relatively clean transitions, including $\mathrm{CH}_{2} \mathrm{OHCHO} 9(1,8)-8(2,7), \mathrm{CH}_{3} \mathrm{OCHO}$ 7(2,5)-6(2,4)E, t-HCOOH 4(0,4)-3(0,3), $\mathrm{C}_{2} \mathrm{H}_{5} \mathrm{OH} 4(1,4)-3(0,3)$ and $\mathrm{CH}_{3} \mathrm{NH}_{2} 1(1,0)-1(0,1), \mathrm{CH}_{3} \mathrm{OCH}_{3} 2(2,1)-2(1,2) \mathrm{EE}, \mathrm{C}_{2} \mathrm{H}_{5} \mathrm{CN}$ 10(4,7)-9(4,6) and $\mathrm{H}_{2} \mathrm{NCH}_{2} \mathrm{CN} 10(0,10)-9(3,6)$, are presented in Figs 2 and 3. The positions of Sgr B2(N) and Sgr B2(M) are indicated with crosses. The peak integrated intensities on the $\mathrm{T}_{m b}$ scale are given in the caption. We can see from Figs 2 and 3 that some molecular line emissions concentrate on Sgr B2(N) and Sgr $\mathrm{B} 2(\mathrm{M})$, and some molecular line emissions extend to the eastern part of Sgr B2. Based on their spatial distributions, molecules can be classified as either 'extended', those detected not only in Sgr B2(N) and Sgr B2(M), or 'compact', those only detected toward or near to Sgr B2(N) and Sgr B2(M).

The excitation analysis of glycolaldehyde emissions at millimetre and centimetre wavelengths in Sgr B2(N) indicates that a single temperature component was unable to reproduce all the observed spectra (Hollis et al. 2004). Thus, two temperature components were invoked: a 'warm' extended glycolaldehyde envelope surrounded 
Table 1. Identified transitions and their observed parameters in Sgr B2(N).

\begin{tabular}{|c|c|c|c|c|c|c|c|c|}
\hline Species & Transitions & $\begin{array}{l}\text { Rest freq. } \\
(\mathrm{GHz})\end{array}$ & $\begin{array}{c}E_{\mathrm{u}} \\
(\mathrm{K})\end{array}$ & $\begin{array}{l}\mu^{2} \mathrm{~S} \\
\left(\mathrm{D}^{2}\right)\end{array}$ & $\begin{array}{c}T_{m b} \\
(\mathrm{mK})\end{array}$ & $\begin{array}{c}V_{\mathrm{LSR}} \\
\left(\mathrm{km} \mathrm{s}^{-1}\right)\end{array}$ & $\begin{array}{c}\Delta V \\
\left(\mathrm{~km} \mathrm{~s}^{-1}\right)\end{array}$ & $\underset{\left(\mathrm{mK} \mathrm{km} \mathrm{s}^{-1}\right)}{\int T_{m b} \mathrm{~d} v}$ \\
\hline $\mathrm{t}-\mathrm{HCOOH}^{*}$ & $4(0,4)-3(0,3)$ & $89.57917(1 \mathrm{e}-6)$ & 10.76 & 8.077 & $\sim 100$ & $\sim 64$ & $\sim 20$ & $\sim 2000$ \\
\hline $\mathrm{C}_{2} \mathrm{H}_{5} \mathrm{CN}, v=0$ & $10(9,1)-9(9,0)$ & $89.584987(5 e-5)$ & 109.32 & 28.16 & blended & - & - & - \\
\hline $\mathrm{C}_{2} \mathrm{H}_{5} \mathrm{CN}, v=0$ & $10(9,2)-9(9,1)$ & $89.584987(5 e-5)$ & 109.32 & 28.16 & blended & - & - & - \\
\hline $\mathrm{C}_{2} \mathrm{H}_{5} \mathrm{CN}, v=0^{*}$ & $10(4,7)-9(4,6)$ & $89.590028(3 e-6)$ & 41.44 & 124.52 & $160(45)$ & $63.0(.6)$ & $22.0(1.4)$ & $3520(610)$ \\
\hline $\mathrm{C}_{2} \mathrm{H}_{5} \mathrm{CN}, v=0$ & $10(4,6)-9(4,5)$ & $89.591013(3 e-6)$ & 41.44 & 124.52 & $160(45)$ & 63.0(.6) & $22.0(1.4)$ & $3520(610)$ \\
\hline $\mathrm{C}_{2} \mathrm{H}_{5} \mathrm{CN}, v=0$ & $10(3,8)-9(3,7)$ & $89.628485(3 e-6)$ & 33.66 & 134.89 & $200(10)$ & $65.0(1.4)$ & $21.9(4.0)$ & $4666(654)$ \\
\hline $\mathrm{C}_{2} \mathrm{H}_{5} \mathrm{CN}, v 20=1$ & $10(8,3)-9(8,2)$ & $89.643412(2 \mathrm{e}-6)$ & 630.76 & 52.43 & blended & & & \\
\hline $\mathrm{C}_{2} \mathrm{H}_{5} \mathrm{CN}, v 20=1$ & $10(4,7)-9(4,6)$ & $89.661002(5 e-5)$ & 577.94 & 122.32 & $30(3)$ & $67.2(.4)$ & $7.0(1.2)$ & 211(57) \\
\hline $\mathrm{C}_{2} \mathrm{H}_{5} \mathrm{CN}, v=0$ & $10(3,7)-9(3,6)$ & $89.684710(3 e-6)$ & 33.66 & 134.88 & $134(3)$ & $60.9(2.6)$ & $26.8(2.6)$ & $3821(236)$ \\
\hline $\mathrm{CH}_{3} \mathrm{OCH}_{3}, v=0$ & $2(2,1)-2(1,2) \mathrm{AE}$ & $89.697737(1 \mathrm{e}-6)$ & 8.36 & 8.475 & $91(8)$ & $66.5(2.6)$ & $18.4(2.6)$ & 1783(158) \\
\hline $\mathrm{CH}_{3} \mathrm{OCH}_{3}, v=0^{*}$ & $2(2,1)-2(1,2) \mathrm{EE}$ & $89.699809(1 e-6)$ & 8.36 & 22.31 & $50(8)$ & $64.2(2.6)$ & $15.1(2.6)$ & 797(158) \\
\hline $\mathrm{CH}_{3} \mathrm{OCH}_{3}, v=0$ & $2(2,1)-2(1,2) \mathrm{AA}$ & $89.702829(2 e-6)$ & 8.36 & 14.131 & $46(8)$ & $65.2(.3)$ & $23.0(2.6)$ & 1131(158) \\
\hline $\mathrm{CH}_{3} \mathrm{OCHO}, v t=1$ & $8(0,8)-7(0,7) \mathrm{A}$ & $89.731695(1 \mathrm{e}-5)$ & 207.83 & 41.664 & $29(6)$ & $64.2(.3)$ & $7.4(.7)$ & 228(19) \\
\hline $\mathrm{C}_{2} \mathrm{H}_{5} \mathrm{CN}, v 20=1$ & $10(3,7)-9(3,6)$ & $89.754402(5 e-5)$ & 570.24 & 132.51 & $43(5)$ & $65.9(.4)$ & $34.3(1.2)$ & $1579(46)$ \\
\hline $\mathrm{H}_{2} \mathrm{NCH}_{2} \mathrm{CN}^{*}$ & $10(0,10)-9(3,6)$ & $89.770285(7 e-6)$ & 19.50 & 66.296 & $25(3)$ & $63.7(0.7)$ & $14.5(1.6)$ & $377(40)$ \\
\hline $\mathrm{t}-\mathrm{HCOOH}$ & $4(2,3)-3(2,2)$ & $89.861484(2 \mathrm{e}-6)$ & 23.51 & 5.93 & $50(3)$ & $66.9(.2)$ & $18.5(.6)$ & $987(26)$ \\
\hline $\mathrm{CH}_{2} \mathrm{OHCHO}^{*}$ & $9(1,8)-8(2,7)$ & $89.868639(6 e-6)$ & 26.43 & 20.565 & $17(3)$ & $61.5(2.6)$ & $18.6(2.6)$ & $338(41)$ \\
\hline $\mathrm{t}-\mathrm{HCOOH}$ & $4(3,2)-3(3,1)$ & $89.94821(2 \mathrm{e}-6)$ & 35.09 & 3.536 & blended & & & \\
\hline $\mathrm{CH}_{3} \mathrm{NH}_{2}{ }^{*}$ & 1(1)A $1-1(0) \mathrm{A} 2$ & $89.956068(2 \mathrm{e}-5)$ & 6.45 & $1.5^{a}$ & $31(3)$ & $67.8(1.3)$ & $30.3(2.8)$ & $982(87)$ \\
\hline $\mathrm{CH}_{3} \mathrm{CH}_{2} \mathrm{C}^{13} \mathrm{~N}$ & $10(2,8)-9(2,7)$ & $89.992750(1.2 \mathrm{e}-6)$ & 28.10 & 139.763 & blended & & & \\
\hline $\mathrm{g}-\mathrm{C}_{2} \mathrm{H}_{5} \mathrm{OH}$ & $13(1,12)-13(1,13), \mathrm{g}+$ & $90.0077(5 e-5)$ & 135.11 & 13.99 & $28(3)$ & $64.0(.5)$ & $6.2(1.2)$ & $188(53)$ \\
\hline${ }^{13} \mathrm{CH}_{2} \mathrm{CHCN}$ & $10(1,10)-9(1,9)$ & $90.032588(1.2 \mathrm{e}-6)$ & 25.92 & 432.232 & blended & & & \\
\hline $\mathrm{CH}_{3}^{13} \mathrm{CH}_{2} \mathrm{CN}, v=0$ & $10(2,8)-9(2,7)$ & $90.079288(1.3 e-6)$ & 28.00 & 139.77 & $24(3)$ & $66.3(6)$ & $17.7(1.3)$ & $457(29)$ \\
\hline $\mathrm{C}_{2} \mathrm{H}_{5} \mathrm{OH}^{*}$ & $4(1,4)-3(0,3)$ & $90.11761(2.8 \mathrm{e}-6)$ & 9.36 & 5.21 & $266(9)$ & $68.5(.1)$ & $22.4(.2)$ & $6330(37)$ \\
\hline $\mathrm{CH}_{3} \mathrm{OCHO}^{*}$ & $7(2,5)-6(2,4) E$ & $90.145634(2.4 \mathrm{e}-5)$ & 19.68 & 34.275 & $86(7)$ & $65.7(.1)$ & $16.7(.4)$ & $1523(28)$ \\
\hline $\mathrm{CH}_{3} \mathrm{OCHO}$ & $7(2,5)-6(2,4) \mathrm{A}$ & $90.156473(1 \mathrm{e}-5)$ & 19.67 & 34.289 & $80(7)$ & $66.3(.4)$ & $13.7(.9)$ & $1166(65)$ \\
\hline
\end{tabular}

Note.*: Lines used for mapping. Col. (1): chemical formula; Col. (2): transition quantum numbers; Col. (3): rest frequency; Col. (4): upper-state energy level (K); Col. (5): dipole-weighted transition dipole matrix elements; Col. (6): peak temperature; Col. (7): centroid velocity; Col. (8): FWHM linewidth; Col. (9): integrated intensity. ${ }^{a}: \mu^{2} \mathrm{~S}$ is taken from Motiyenko et al. (2014) for $\mathrm{CH}_{3} \mathrm{NH}_{2}$.

by a 'cold' glycolaldehyde halo (Hollis et al. 2004). The $89 \mathrm{GHz}$ transitions with a higher upper-level energy $(26.43 \mathrm{~K})$ are regarded as coming from the 'warm' phase, while transitions with a lower upper-level energy $(1.2 \mathrm{~K})$ are regarded as coming from the 'cold' phase. The 'cold' glycolaldehyde was found to be widespread around the Sgr B2 complex (Li et al. 2017). Fig. 2(a) shows a contour map of the velocity-integrated intensities of the 'warm' glycolaldehyde emission obtained with the ARO $12 \mathrm{~m}$ telescope overlaid on the 'cold' glycolaldehyde emission acquired with the Shanghai Tianma $65 \mathrm{~m}$ telescope in greyscale ( $\mathrm{Li}$ et al. 2017). The half-power beamwidths were $\sim 70$ arcsec and $\sim 77$ arcsec for the ARO $12 \mathrm{~m}$ observations and $65 \mathrm{~m}$ observations, respectively. The 'cold' glycolaldehyde is distributed across $800 \operatorname{arcsec}(\sim 33 \mathrm{pc}$ in diameter), which is substantially more extended than the 'warm' glycolaldehyde. The 'warm' glycolaldehyde emission shows several peaks, including the north cloud, the peak near Sgr B2(M), and the ridge west of Sgr B2(N). A weak 'warm' glycolaldehyde emission at $89 \mathrm{GHz}$ was also detected toward the eastern component of the Sgr B2 complex.

According to BIMA observations (Hollis et al. 2001), glycolaldehyde is greatly extended in comparison to the ethyl cyanide and dimethyl ether, which are largely confined to the Large Molecule Heimat source (LMH) (Snyder, Kuan \& Miao 1994; Miao et al. 1995), so these molecules were classified as either 'extended' or 'compact' here based on the half-power radius of the spatial distributions in Figs 2 and 3. Fig. 4 shows variation of the normalized integrated intensity of observed transitions with the distance to Sgr $\mathrm{B} 2(\mathrm{~N})$ for sampling points with $\triangle \mathrm{RA}=0$. The normalized intensity is obtained by dividing the integrated intensity by the maximum integrated intensity of molecule. We change the declination and take a measurement at constant RA $(\Delta \mathrm{RA}=0)$. FWHM, the full width at half maximum, is always used to evaluate the linewidth of spectral lines. Thus we selected 50 percent as a metric to evaluate the distribution of molecules. We can see from Fig. 4 that the normalized intensity of $\mathrm{CH}_{3} \mathrm{OCH}_{3}, \mathrm{C}_{2} \mathrm{H}_{5} \mathrm{CN}$ and $\mathrm{H}_{2} \mathrm{NCH}_{2} \mathrm{CN}$ quickly decrease below 50 per cent for sampling points away from Sgr B2(N). The half-power radii of these molecules are smaller than the sampling interval ( $1 \mathrm{arcmin})$. Thus these three molecules are classified as 'compact' molecules. $\mathrm{H}_{2} \mathrm{NCH}_{2} \mathrm{CN}$ was only detected in Sgr B2(N) in our observations; thus it is also classified as a 'compact' molecule. On the other hand, the normalized intensities of $\mathrm{CH}_{2} \mathrm{OHCHO}, \mathrm{CH}_{3} \mathrm{OCHO}$, $\mathrm{t}-\mathrm{HCOOH}, \mathrm{C}_{2} \mathrm{H}_{5} \mathrm{OH}$ and $\mathrm{CH}_{3} \mathrm{NH}_{2}$ vary slowly with the distance to $\mathrm{Sgr} \mathrm{B} 2(\mathrm{~N})$; thus these molecules are classified as 'extended' molecules. In order to investigate whether only the strongest lines are extended, the 'extended' line profiles were coloured red, while the 'compact' line profiles were coloured blue in the spectra of Sgr B2(N) (Fig. 1, upper panel). As is shown in the upper panel of Fig. 1, the strongest emissions come from $\mathrm{C}_{2} \mathrm{H}_{5} \mathrm{CN}, \mathrm{C}_{2} \mathrm{H}_{5} \mathrm{OH}$, t-HCOOH and $\mathrm{CH}_{3} \mathrm{OCH}_{3}$. Among them, the $\mathrm{C}_{2} \mathrm{H}_{5} \mathrm{CN}$ and $\mathrm{CH}_{3} \mathrm{OCH}_{3}$ emissions are confined to Sgr B2(N) and Sgr B2(M). Thus it is sure that not all the strongest lines are the most extended. It is noted that $\mathrm{CH}_{2} \mathrm{OHCHO}$ peaks away from $\mathrm{Sgr}$ $\mathrm{B} 2(\mathrm{~N})$, which seems to differ from most COMs presented in this paper. The line blending makes it difficult to obtain the intensity of $\mathrm{CH}_{2} \mathrm{OHCHO}$ accurately. We have mapped $\mathrm{CH}_{2} \mathrm{OHCHO}$ around $\mathrm{Sgr}$ B2 with the IRAM $30 \mathrm{~m}$ telescope with better spatial and spectral resolution to further investigate this issue. The preliminary result is consistent with the ARO $12 \mathrm{~m}$ result presented here. A detailed 
(a)

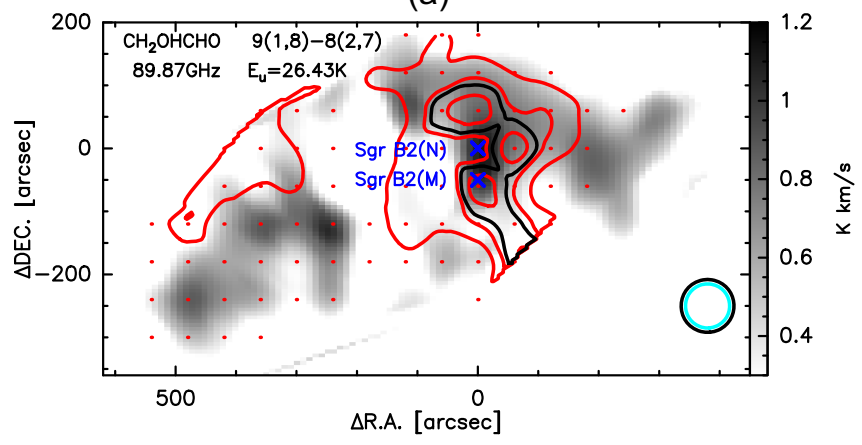

(c)

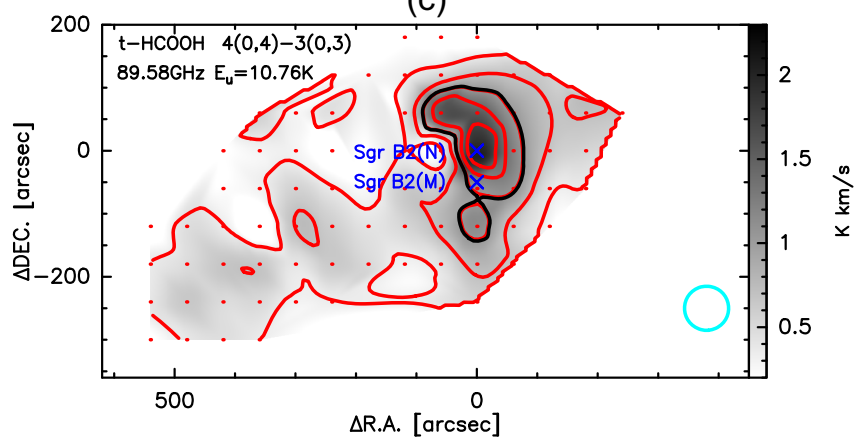

(b)

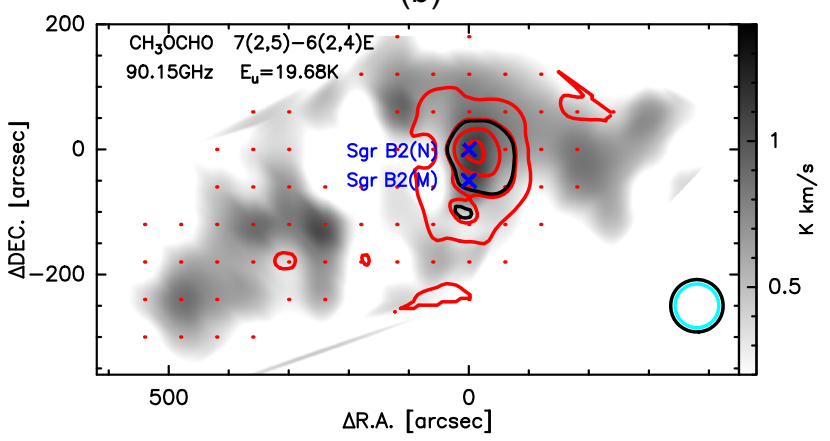

(d)

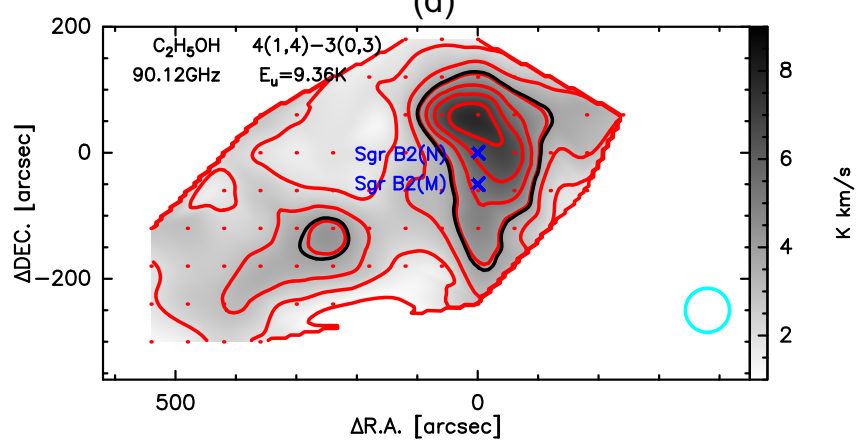

(e)

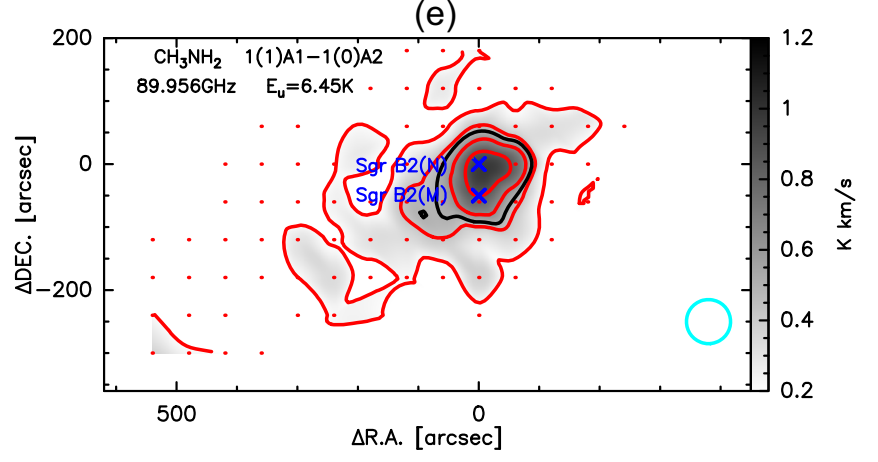

Figure 2. (a) Velocity-integrated intensity map of 'warm' glycolaldehyde $\mathrm{CH}_{2} \mathrm{OHCHO} 9(1,8)-8(2,7)$ (contours) overlaid on a velocity-integrated intensity map of 'cold' glycolaldehyde $\mathrm{CH}_{2} \mathrm{OHCHO} 1(1,0)-1(0,1)$ observed at $13 \mathrm{GHz}$ (Li et al. 2017) in greyscale. The contours are from $3 \sigma$ increasing in steps of $3 \sigma$, which corresponds to $0.21 \mathrm{~K} \mathrm{~km} \mathrm{~s}^{-1}$. The 70 arcsec FWHM ARO beam at $90 \mathrm{GHz}$ is shown by a cyan circle, while the 77 arcsec FWHM TMRT beam at $13 \mathrm{GHz}$ is shown by a black circle. (b) Velocity-integrated intensity map of $\mathrm{CH}_{3} \mathrm{OCHO} 7(2,5)-6(2,4) \mathrm{E}$ (contours) overlaid on a velocity-integrated intensity map of 'cold' glycolaldehyde $\mathrm{CH}_{2} \mathrm{OHCHO} 1(1,0)-1(0,1)$ observed at $13 \mathrm{GHz}$ ( $\mathrm{Li}$ et al. 2017) in greyscale. The contours are from $5 \sigma$ increasing in steps of $5 \sigma$, which corresponds to $0.35 \mathrm{~K} \mathrm{~km} \mathrm{~s}^{-1}$. The 70 arcsec FWHM ARO beam at $90 \mathrm{GHz}$ is shown by a cyan circle, while the 77 arcsec FWHM TMRT beam at $13 \mathrm{GHz}$ is shown by a black circle. (c) Velocity-integrated intensity map of t-HCOOH 4(0,4)-3(0,3). The contours are from $5 \sigma$ increasing in steps of $5 \sigma$, which corresponds to $0.35 \mathrm{~K} \mathrm{~km} \mathrm{~s}^{-1}$. (d) Velocity-integrated intensity map of $\mathrm{C}_{2} \mathrm{H}_{5} \mathrm{OH} 4(1,4)-3(0,3)$. The red contours started from and have steps of $1 \mathrm{~K}$ $\mathrm{km} \mathrm{s}^{-1}$. (e) Velocity-integrated intensity map of $\mathrm{CH}_{3} \mathrm{NH}_{2} 1(1,0)-1(0,1)$. The contours are from $3 \sigma$ increasing in steps of $3 \sigma$, which corresponds to $0.21 \mathrm{~K}$ km $\mathrm{s}^{-1}$. The black contours represent 50 per cent of the map peaks, while dots stand for the sampling points in each sub-plot. The 70 arcsec FWHM ARO beam at $90 \mathrm{GHz}$ is shown by a cyan circle in each sub-plot.

chemical model is needed to explain the spatial distribution of glycolaldehyde in this region.

\section{1 'Extended' molecules}

\subsubsection{Glycolaldehyde $\mathrm{CH}_{2} \mathrm{OHCHO}$}

$\mathrm{CH}_{2} \mathrm{OHCHO}$, which is a sugar-related molecule, can react with propenal to form ribose - a central constituent of RNA (Sharma et al. 2016). $\mathrm{CH}_{2} \mathrm{OHCHO}$ was first detected in $\mathrm{Sgr} \mathrm{B} 2(\mathrm{~N})$ based on the emission spectra in the millimetre-wave range observed with the NRAO $12 \mathrm{~m}$ telescope (Hollis et al. 2000). The $\mathrm{CH}_{2} \mathrm{OHCHO}$ $8(0,8)-7(1,7)$ emission at $89 \mathrm{GHz}$ was regarded as coming from 'warm' glycolaldehyde, while the $13 \mathrm{GHz}$ emission was regarded as coming from 'cold' glycolaldehyde (Hollis et al. 2004). The distribution of 'warm' glycolaldehyde is extended and seems to be composed of several emission cores (see Fig. 2). Sensitive interferometric observations are needed to resolve and study these 
(a)

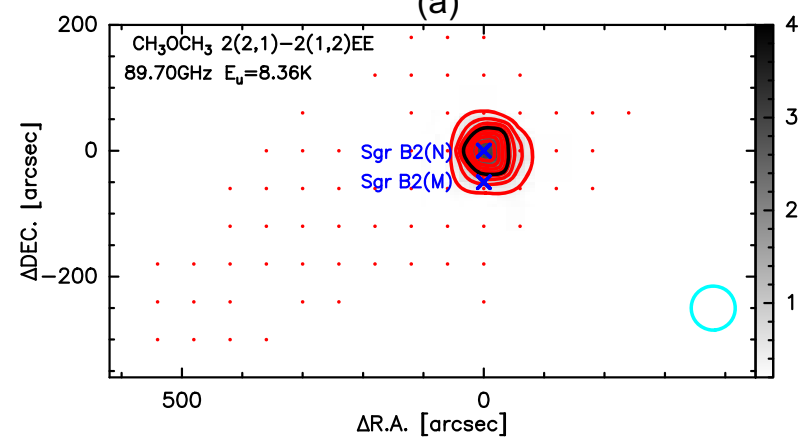

(b)

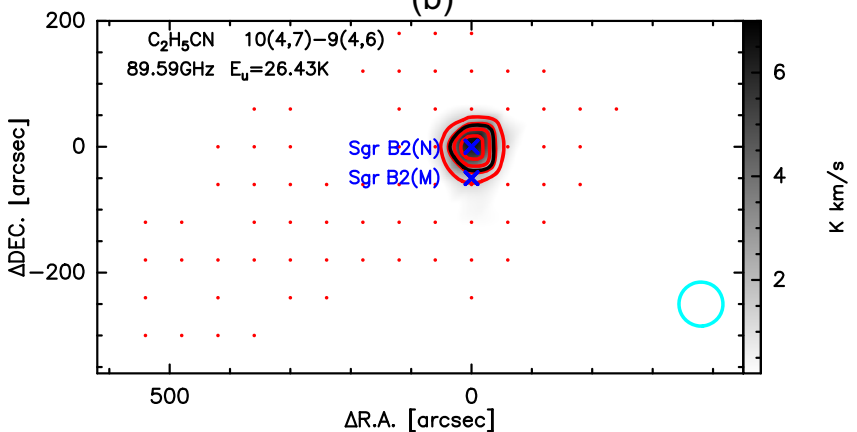

(c)

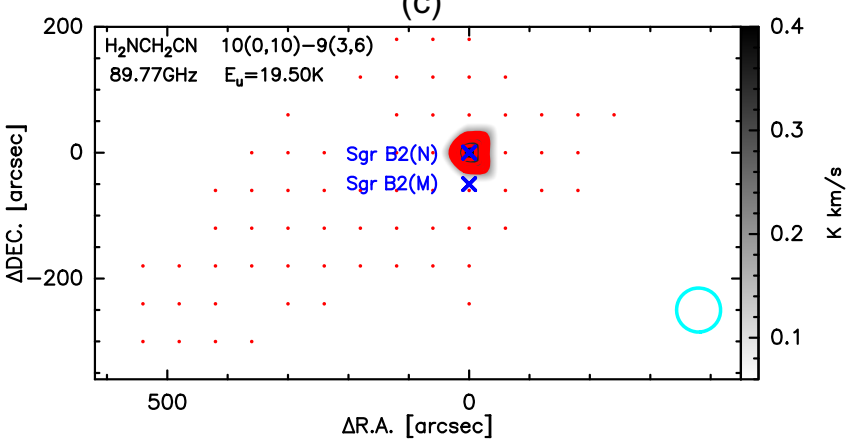

Figure 3. (a) Velocity-integrated intensity map of $\mathrm{CH}_{3} \mathrm{OCH}_{3} 2(2,1)-2(1,2) \mathrm{EE}$ observed with the ARO $12 \mathrm{~m}$ telescope. The contours are from $10 \sigma$ increasing in steps of $10 \sigma$, which corresponds to $0.7 \mathrm{~K} \mathrm{~km} \mathrm{~s}^{-1}$. (b) Velocity-integrated intensity map of $\mathrm{C}_{2} \mathrm{H}_{5} \mathrm{CN} 10(4,7)-9(4,6)$ observed with the ARO $12 \mathrm{~m}$ telescope. The contours are from $20 \sigma$ increasing in steps of $20 \sigma$, which corresponds to $1.4 \mathrm{~K} \mathrm{~km} \mathrm{~s}^{-1}$. (c) Velocity-integrated intensity map of $\mathrm{H}_{2} \mathrm{NCH}_{2} \mathrm{CN} 10(0,10)-9(3,6)$ observed with the ARO $12 \mathrm{~m}$ telescope. The contours are from $5 \sigma$ increasing in steps of $\sigma$, which corresponds to $0.04 \mathrm{~K}$ km s ${ }^{-1}$. The black contours represent 50 per cent of the map peaks. Dots stand for the sampling points. The 70 arcsec FWHM ARO beam at 90 GHz is shown by a cyan circle in each sub-plot.

cores in detail. The 'cold' glycolaldehyde has been found to be widespread around the Sgr B2 complex (Li et al. 2017). The different spatial distribution of 'warm' and 'cold' glycolaldehyde seems to be caused by different excitation conditions, including the temperature and density of molecular gas.

\subsubsection{Methyl formate $\mathrm{CH}_{3} \mathrm{OCHO}$}

ARO observations indicate that the spatial distribution of methyl formate emission significantly differs from both the 'warm' and the 'cold' glycolaldehyde. It seems to peak toward Sgr B2(N) and Sgr B2(M), and decrease toward the surroundings. Weak emission was seen at the eastern part of the Sgr B2 complex.

\subsubsection{Formic acid $t$ - $\mathrm{HCOOH}$}

$\mathrm{t}-\mathrm{HCOOH}$ is a key organic molecule as the carboxyl group $(\mathrm{C}(=\mathrm{O}) \mathrm{OH})$ is one of the main functional groups of amino acids (the structural units of proteins). This species is involved in a chemical route leading to glycine, the simplest amino acid (Redondo, Largo \& Barrientos 2015). ARO observations indicate that the spatial distribution of $\mathrm{t}-\mathrm{HCOOH}$ extends to the eastern component and peaks at Sgr B2(N).

\subsubsection{Ethanol $\mathrm{C}_{2} \mathrm{H}_{5} \mathrm{OH}$}

$\mathrm{C}_{2} \mathrm{H}_{5} \mathrm{OH}$ was first detected toward Sgr B2 (Zuckerman et al. 1975). This molecular was found to be present only in the dense $\left(\geq 10^{6}\right.$ $\left.\mathrm{cm}^{-3}\right)$ and hot $(\geq 100 \mathrm{~K})$ cores associated with newly formed massive stars (Martín-Pintado et al. 2001; Bisschop et al. 2007; Qin et al. 2010; Widicus Weaver et al. 2017). Martín-Pintado et al. (2001) found that $\mathrm{C}_{2} \mathrm{H}_{5} \mathrm{OH}$ emission in $\mathrm{Sgr} \mathrm{B} 2$ is widespread. They proposed that $\mathrm{C}_{2} \mathrm{H}_{5} \mathrm{OH}$ formed in grains and was released to the gas phase by shocks in the last $\sim 10^{5} \mathrm{yr}$. The results presented here provide further evidence for the presence of extended ethanol around Sgr B2. The $\mathrm{C}_{2} \mathrm{H}_{5} \mathrm{OH}$ emission peaks at the north of Sgr B2(N).

\subsubsection{Methylamine $\mathrm{CH}_{3} \mathrm{NH}_{2}$}

$\mathrm{CH}_{3} \mathrm{NH}_{2}$ was first detected in Sgr B2(OH) (Kaifu et al. 1974). It was also detected in the southern region of Sgr B2 (Halfen, Ilyushin \& Ziurys 2013). $\mathrm{CH}_{3} \mathrm{NH}_{2}$ is thought to be a potential interstellar precursor to the amino acid glycine, $\mathrm{NH}_{2} \mathrm{CH}_{2} \mathrm{COOH}$. The reaction of $\mathrm{CH}_{3} \mathrm{NH}_{2}$ with $\mathrm{CO}_{2}$ in water ice has been shown to yield $\mathrm{NH}_{2} \mathrm{CH}_{2} \mathrm{COOH}$ after UV irradiation (Bossa et al. 2009; Lee et al. 2009). Our observations indicate that $\mathrm{CH}_{3} \mathrm{NH}_{2}$ emission peaks at Sgr B2(N), and extends to the eastern part of Sgr B2 complex, which is in agreement with previous results.

\section{2 'Compact' molecules}

\subsubsection{Dimethyl ether $\mathrm{CH}_{3} \mathrm{OCH}_{3}$}

The $\mathrm{CH}_{3} \mathrm{OCH}_{3}$ emission is detected toward Sgr B2(N) and a few points near to $\mathrm{Sgr} \mathrm{B} 2(\mathrm{~N})$. Though $\mathrm{CH}_{3} \mathrm{OCH}_{3}$ is an isomer of $\mathrm{C}_{2} \mathrm{H}_{5} \mathrm{OH}$, the distributions of these two molecules are strikingly different, implying that they have different chemical routes, which 

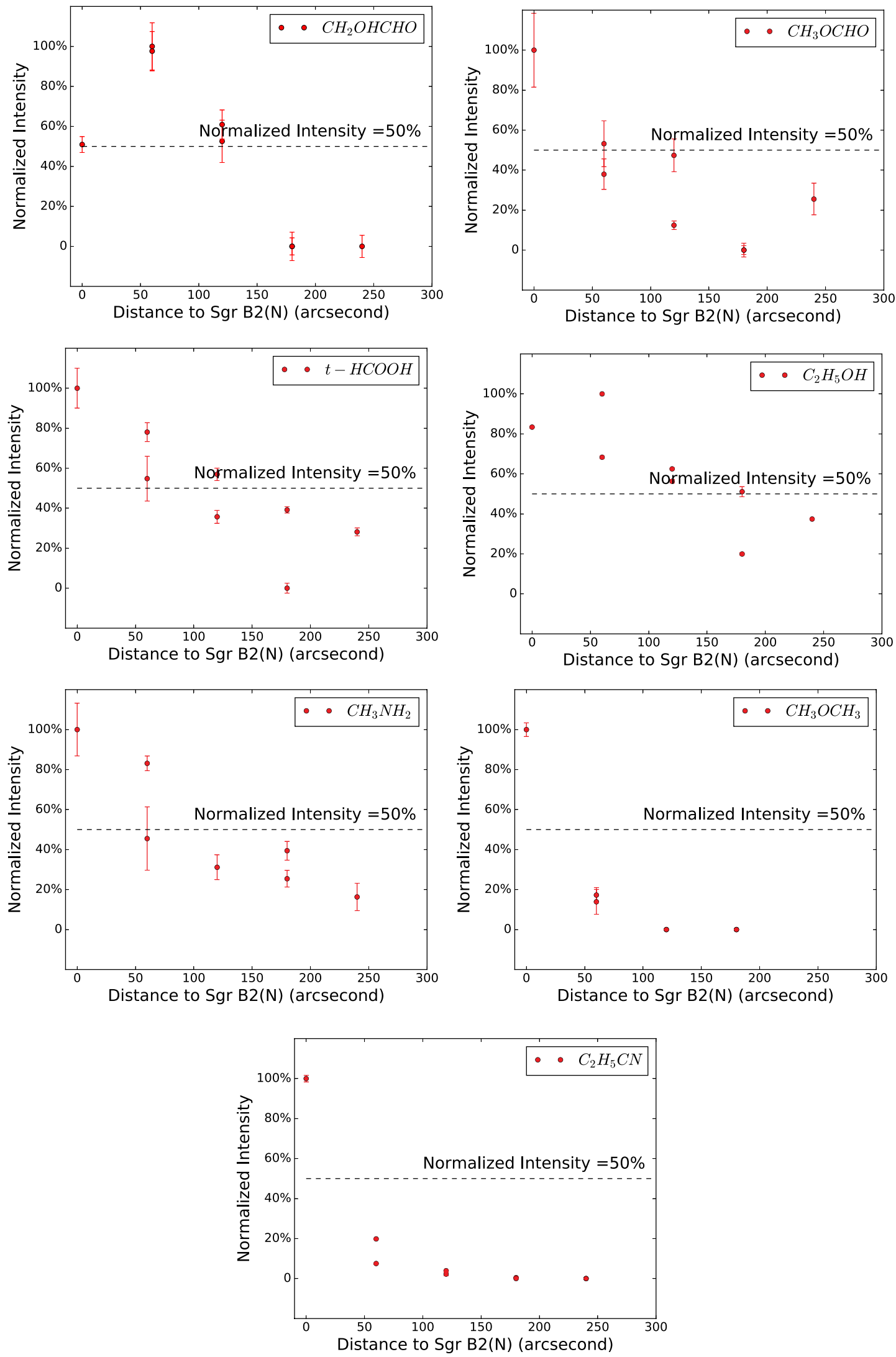

Figure 4. Variation of the normalized intensity of observed transitions versus the distance to Sgr $\mathrm{B} 2(\mathrm{~N})$ for sampling points with $\Delta \mathrm{RA}=0$. The normalized intensity is obtained by dividing the integrated intensity by the maximum integrated intensity of the molecule. We change declination and take a measurement at constant $\mathrm{RA}(\triangle \mathrm{RA}=0)$. 
is consistent with observations in W51 (Rong et al. 2016). As $\mathrm{C}_{2} \mathrm{H}_{5} \mathrm{OH}$ is thought to be produced by grain-surface reactions, $\mathrm{CH}_{3} \mathrm{OCH}_{3}$ is likely to form via gas-phase chemistry. The spatial distribution of $\mathrm{CH}_{3} \mathrm{OCH}_{3}$ also differs from that of $\mathrm{CH}_{3} \mathrm{OCHO}$, which seems to be different from observations in massive starforming regions in the Galactic disc. A striking similarity was found in the spatial distribution, temperature and column densities of these two molecules in star-forming regions in the Galactic disc (Brouillet et al. 2013; Jaber et al. 2014; Rong et al. 2016). $\mathrm{CH}_{3} \mathrm{OCH}_{3}$ has been proposed as a precursor molecule to $\mathrm{CH}_{3} \mathrm{OCHO}$ (Balucani, Ceccarelli \& Taquet 2015). The obvious difference in spatial distribution of $\mathrm{CH}_{3} \mathrm{OCH}_{3}$ and $\mathrm{CH}_{3} \mathrm{OCHO}$ observed in $\mathrm{Sgr}$ $\mathrm{B} 2$ suggests that the formation mechanism of these two molecules might differ in the Galactic centre and Galactic disc sources.

\subsubsection{Ethyl cyanide $\mathrm{C}_{2} \mathrm{H}_{5} \mathrm{CN}$}

Previous observations of $\mathrm{C}_{2} \mathrm{H}_{5} \mathrm{CN}$ in $\mathrm{Sgr} \mathrm{B} 2$ have shown that this molecule is confined to a small region, and is likely to be located in the hot dense core of the star-forming region (Miao \& Snyder 1997). Our mapping results show that $\mathrm{C}_{2} \mathrm{H}_{5} \mathrm{CN}$ was only detected toward Sgr B2(N) and at four points near to Sgr B2(N), which agree with previous studies.

\subsubsection{Amino acetonitrile $\mathrm{H}_{2} \mathrm{NCH}_{2} \mathrm{CN}$}

$\mathrm{H}_{2} \mathrm{NCH}_{2} \mathrm{CN}$ was first detected in Sgr B2(N) (Belloche et al. 2008). The source size was estimated to be about 2 arcmin FWHM. No evidence for colder more extended emission was found. Amino acetonitrile may well be a direct precursor of glycine. In our observations, $\mathrm{H}_{2} \mathrm{NCH}_{2} \mathrm{CN}$ was only detected toward Sgr B2(N).

\subsection{Column densities}

According to IRAM 30 m observations of COMs (Requena-Torres et al. 2006), $T_{\text {rot }}$ derived from $\mathrm{C}_{2} \mathrm{H}_{5} \mathrm{OH}$ is $72.6 \mathrm{~K}$ for $\mathrm{Sgr} \mathrm{B} 2(\mathrm{~N})$, and $55.4 \mathrm{~K}$ for $\mathrm{Sgr} \mathrm{B} 2(\mathrm{M}) . T_{\text {rot }}$ derived from $\mathrm{C}_{2} \mathrm{H}_{5} \mathrm{OH}$ ranges from 9 $14 \mathrm{~K}$ for other Galactic centre clouds; $T_{\text {rot }}$ derived from $\mathrm{CH}_{3} \mathrm{OCHO}$ ranges from $8-16 \mathrm{~K}$ for other Galactic centre clouds. Assuming an excitation temperature of $50 \mathrm{~K}$ for Sgr B2(N) and Sgr B2(M), and $14 \mathrm{~K}$ for other positions, we calculate the total column densities of 'warm' $\mathrm{CH}_{2} \mathrm{OHCHO}, \mathrm{CH}_{3} \mathrm{OCHO}, \mathrm{C}_{2} \mathrm{H}_{5} \mathrm{OH}$ and t- $\mathrm{HCOOH}$ for the $i, j$ th grid in the position-position space $N_{i j}$ with the expression reported by Hollis et al. (2004):

$N_{i j}=\frac{3 k Q \mathrm{e}^{E_{u} / k T_{S}}}{8 \pi^{3} v S \mu^{2}} \times \frac{\frac{1}{2} \sqrt{\frac{\pi}{\ln 2}} \frac{\Delta T_{A}^{*} \Delta V}{\eta_{B}}}{1-\frac{\mathrm{e}^{h \nu / k T_{S}-1}}{\mathrm{e}^{h \nu / K T_{\mathrm{bg}}-1}}}$,

in which $k$ is the Boltzmann constant in $\operatorname{erg} \mathrm{K}^{-1}, \frac{\Delta T_{A}^{*} \Delta V}{\eta_{B}}$ is the observed line integrated intensity in $\mathrm{K} \mathrm{Km} \mathrm{s}^{-1}$, $v$ is the frequency of the transition in $\mathrm{Hz}$, and $S \mu^{2}$ is the product of the total torsionrotational line strength and the square of the electric dipole moment. $T_{S}$ and $T_{\mathrm{bg}}(=2.73 \mathrm{~K})$ are the excitation temperature and background brightness temperature, respectively. $E_{\mathrm{u}} / k$ is the upper-level energy in $\mathrm{K}$. The partition function, $Q$, was estimated by fitting the partition function at different temperatures given in CDMS (Müller et al. 2005). The values of $E_{\mathrm{u}} / k$ and $S \mu^{2}$ are also taken from CDMS.

Because of the effects of beam dilution, we did not calculate the column densities of 'compact' molecules like $\mathrm{C}_{2} \mathrm{H}_{5} \mathrm{CN}$ and
$\mathrm{CH}_{3} \mathrm{OCH}_{3}$. In the discussion below, we focus only on oxygenbearing molecules showing extended emission, including 'warm' $\mathrm{CH}_{2} \mathrm{OHCHO}, \mathrm{CH}_{3} \mathrm{OCHO}, \mathrm{C}_{2} \mathrm{H}_{5} \mathrm{OH}$ and $\mathrm{t}-\mathrm{HCOOH}$.

We made use of the $\mathrm{H}_{2}$ column density obtained with BGPS $1.1 \mathrm{~mm}$ data (Bally et al. 2010) for abundance estimation of molecules. For each position, we searched for the nearest clump identified with BGPS data, and obtained the corresponding hydrogen column density. The $\mathrm{H}_{2}$ column densities range from $1.1 \times 10^{23}-1.0 \times 10^{25} \mathrm{~cm}^{-2}$ in our observing region, and peaks toward Sgr B2(N) and Sgr B2(M).

\section{DISCUSSIONS}

A comparison between the abundances of ethanol and 'warm' glycolaldehyde relative to that of $\mathrm{N}\left(\mathrm{H}_{2}\right)$ for each position is shown in Fig. 5. We found that $\mathrm{X}\left(\mathrm{CH}_{2} \mathrm{OHCHO}-\right.$ warm $)=0.2 \mathrm{X}\left(\mathrm{C}_{2} \mathrm{H}_{5} \mathrm{OH}\right)$, with a correlation coefficient of 0.96 . This result agrees well with the relation found for star-forming regions in the Galactic disc: L1157-b1 (Lefloch et al. 2017), IRAS 16293-2422 (Jørgensen et al. 2016), IRAS 2A and IRAS 4A (Taquet et al. 2015) (see Fig. 3). The strong correlation between ethanol and 'warm' glycolaldehyde suggests that the 'warm' glycolaldehyde may be chemically related to ethanol. Recently, theoretical studies have shown that a new gasphase scheme of reactions, involving ethanol as a parent molecule, can lead to the formation of glycolaldehyde and formic acid (Skouteris et al. 2018). This model can well explain the abundance correlation between 'warm' glycolaldehyde and ethanol. This model is further supported by the strong correlation between the abundances of ethanol and formic acid relative to that of $\mathrm{N}\left(\mathrm{H}_{2}\right)$ (Fig. 5), with a correlation coefficient of 0.92 . The correlation between the abundances relative to $\mathrm{H}_{2}$ of ethanol, 'warm' glycolaldehyde and formic acid suggests that 'warm' glycolaldehyde and formic acid are chemically related to ethanol and are possibly produced by ethanol via gas-phase reactions (Skouteris et al. 2018). However, we could not exclude the possibility that these molecules are cospatial in the same gas and trace a warm gas phase. Interferometric observations of a large sample of interstellar sources could help to investigate whether ethanol, formic acid and 'warm' glycolaldehyde are co-spatial and constrain their formation pathways (Xue et al. 2019).

Fig. 5 shows a comparison between the abundances relative to $\mathrm{H}_{2}$ of ethanol and 'cold' glycolaldehyde for each position. The abundance of 'cold' glycolaldehyde was observed at the $13 \mathrm{GHz}$ (Li et al. 2017) range from $10^{-11}-10^{-9}$, which is similar to the value for ethanol. We found that $\mathrm{X}_{\mathrm{CH}_{2} \mathrm{OHCHO}-\text { cold }}=0.94 \mathrm{X}_{\mathrm{C}_{2} \mathrm{H}_{5} \mathrm{OH}}$, with a correlation coefficient of 0.98 . Theoretical studies show that gas-phase chemistry cannot produce the observed abundance of ethanol, even under the most favourable conditions (Charnley et al. 1995). Infrared observations also indicate that interstellar ethanol can be formed by grain-surface reactions significantly more efficiently than by gas-phase chemistry (Sandford et al. 1991); thus, ethanol is thought to be a good tracer of dust chemistry. The good correlation $(\sim 1)$ between the abundances of ethanol and 'cold' glycolaldehyde indicate that the 'cold' glycolaldehyde should also be produced by grain-surface reactions. Similar to ethanol, the desorption mechanism for the 'cold' glycolaldehyde should be the large-scale shocks in the Galactic centre region (Martín-Pintado et al. 2001).

Laboratory studies show that glycolaldehyde always forms on icy grains at low temperatures, regardless of UV-photon absorption (i.e. energetic processes), and methyl formate cannot form efficiently in the absence of energetic processes (Chuang et al. 2017). 
(a)

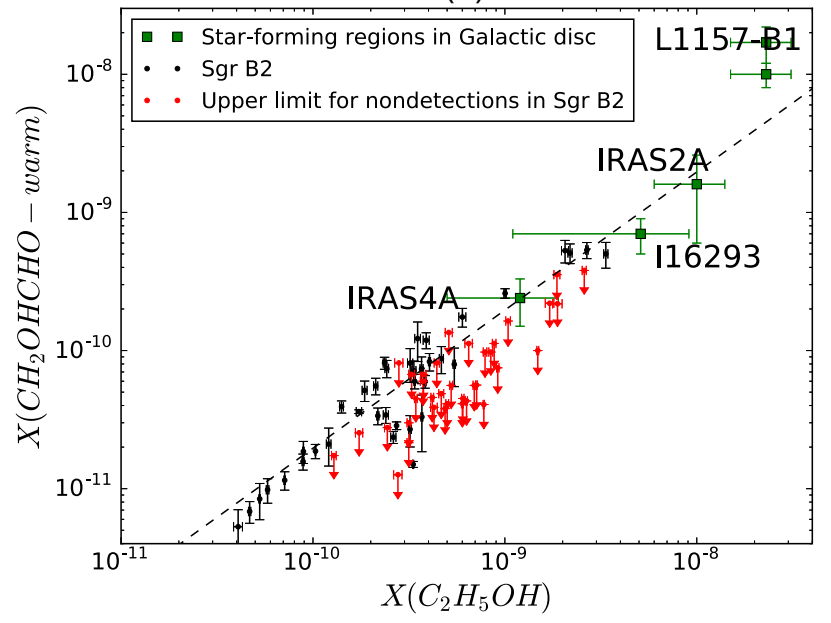

(b)

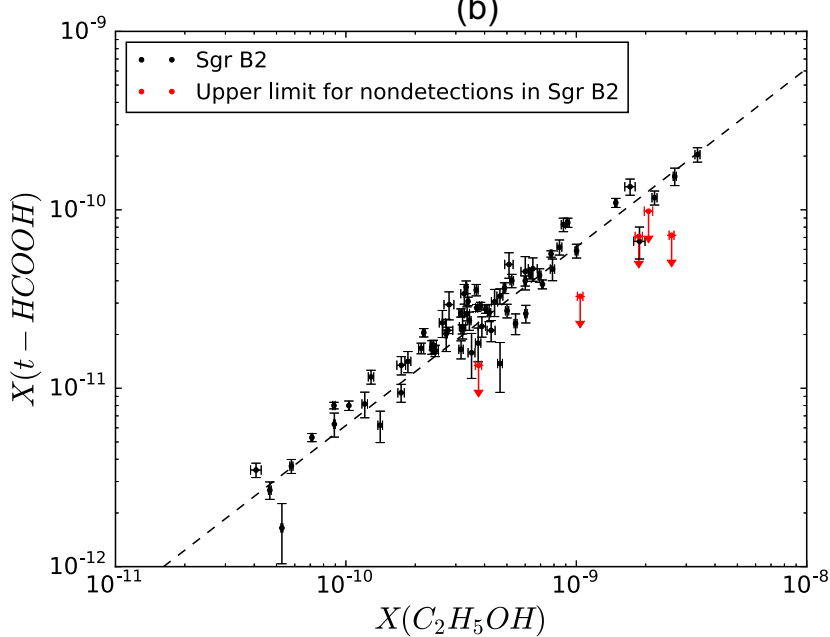

(c)

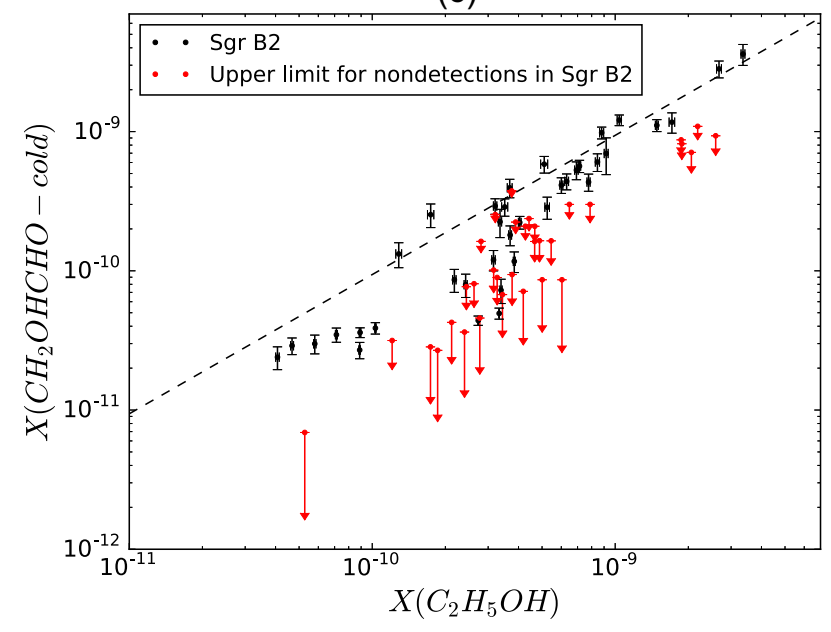

Figure 5. (a) 'Warm' glycolaldehyde abundance as a function of ethanol abundance for different positions in $\mathrm{Sgr} \mathrm{B} 2$. $\mathrm{The}$ relation $\mathrm{X}\left(\mathrm{CH} \mathrm{OHCHO}_{2}-\mathrm{Warm}\right)=$ $0.2 \mathrm{X}\left(\mathrm{C}_{2} \mathrm{H}_{5} \mathrm{OH}\right)$ is marked by the black dashed line. (b) Formic acid abundance as a function of ethanol abundance for different positions in Sgr B2. The relation $\mathrm{X}_{\mathrm{t}-\mathrm{HCOOH}}=0.06 \mathrm{X}_{\mathrm{C}_{2} \mathrm{H}_{5} \mathrm{OH}}$ is marked by the black dashed line. (c) 'Cold' glycolaldehyde abundance as a function of ethanol abundance for different positions in Sgr B2. The relation $\mathrm{X}_{\mathrm{CH}_{2} \mathrm{OHCHO}-\text { cold }}=0.94 \mathrm{X}_{\mathrm{C}_{2} \mathrm{H}_{5} \mathrm{OH}}$ is marked by the black dashed line.

Overabundant methyl formate would mean additional UV photons to low-temperature surface chemistry. In contrast, under-abundant methyl formate would mean that only the low-temperature process is important in the production of complex organic molecules. This view agrees well with the results presented here, in which methyl formate emission is concentrated on Sgr B2(N) and Sgr B2(M), where massive star-forming activity takes place and UV photons are abundant. In contrast, 'cold' glycolaldehyde emission is very widespread and extends to the eastern part of the Sgr B2 complex, where no star-forming activity has been found and the UV radiation should be substantially weaker. Thus, the methyl formate and 'cold' glycolaldehyde near to Sgr B2(N) and Sgr B2(M) likely form on icy grains under UV radiation, while 'cold' glycolaldehyde in regions away from the $\mathrm{H}$ II region likely form on icy grains at low temperature.

\section{SUMMARY}

We have carried out large-scale mapping observations of a series of COMs toward Sgr B2 with the ARO $12 \mathrm{~m}$ telescope.
We obtained large-scale spatial distributions of COMs, including $\mathrm{CH}_{2} \mathrm{OHCHO}, \mathrm{CH}_{3} \mathrm{OCHO}$, t- $\mathrm{HCOOH}, \mathrm{C}_{2} \mathrm{H}_{5} \mathrm{OH}$ and $\mathrm{CH}_{3} \mathrm{NH}_{2}$, $\mathrm{CH}_{3} \mathrm{OCH}_{3}, \mathrm{C}_{2} \mathrm{H}_{5} \mathrm{CN}$, in Sgr B2. The main results of this work include:

(1) The spatial distribution of complex organic molecules can be classified as either 'extended' or 'compact'. The 'extended' molecules include $\mathrm{CH}_{2} \mathrm{OHCHO}, \mathrm{CH}_{3} \mathrm{OCHO}$, t- $\mathrm{HCOOH}, \mathrm{C}_{2} \mathrm{H}_{5} \mathrm{OH}$ and $\mathrm{CH}_{3} \mathrm{NH}_{2}$, while the 'compact' molecules include $\mathrm{CH}_{3} \mathrm{OCH}_{3}$, $\mathrm{C}_{2} \mathrm{H}_{5} \mathrm{CN}$, and $\mathrm{H}_{2} \mathrm{NCH}_{2} \mathrm{CN}$.

(2) The spatial distribution of $\mathrm{CH}_{3} \mathrm{OCHO}$ obviously differs from $\mathrm{CH}_{3} \mathrm{OCH}_{3}$, which has been observed to have similar spatial distribution and column densities to those in star-forming regions of the Galactic disc. These results suggest that the formation mechanisms of these two molecules in the Galactic centre may differ from those in star-forming regions of the Galactic disc.

(3) These 'compact' molecules are likely to be produced under strong UV radiation, while 'extended' molecules are likely to be formed at low temperatures, via gas-phase or grain-surface reactions. 
(4) We found evidence for an overabundance of $\mathrm{CH}_{2} \mathrm{OHCHO}$ compared to that expected from the gas-phase model, which indicates that grain-surface reactions are necessary to explain the origin of $\mathrm{CH}_{2} \mathrm{OHCHO}$ in Sagittarius B2.

Our measurements demonstrate the necessity of grain-surface chemistry for the production of COMs, such as glycolaldehyde in Sgr B2. The results open up an exciting opportunity to study COMs in the circumnuclear disc of starburst galaxies with interferometers including ALMA, the Next Generation Very Large Array (ngVLA) and SKA. Future high-sensitivity interferometric observations possess the potential to probe COM emissions to investigate whether Central Molecular Zone clouds could serve as a template for the nuclei of starburst galaxies in the nearby and distant universe (Kauffman et al. 2017).

\section{ACKNOWLEDGEMENTS}

The Kitt Peak $12 \mathrm{~m}$ telescope is operated by the Arizona Radio Observatory (ARO), Steward Observatory, University of Arizona. We thank the ARO staff for assisting with the observations. This work is partially supported by the National Key R\&D Program of China (No. 2017YFA0402604), the Natural Science Foundation of China (11590780, 11590782, 11590784, U1731237, 11773054, 11725313, 11690024 and 11903038), the Special Funding for Advanced Users, budgeted and administrated by the Center for Astronomical Mega-Science, the Chinese Academy of Sciences (CAMS-CAS) and the CAS 'Light of West China' Program. This work also benefited from the International Space Science Institute (ISSI/ISSI-BJ) in Bern and Beijing, thanks to the funding of the team 'Chemical abundances in the ISM: the litmus test of stellar IMF variations in galaxies across cosmic time' (Principal Investigators DR and Z-YZ).

\section{REFERENCES}

Bally J. et al., 2010, ApJ, 721, 137

Balucani N., Ceccarelli C., Taquet V., 2015, MNRAS, 449, 16

Belloche A., Menten K. M., Comito C., Müller H. S. P., Schilke P., Ott J., Thorwirth S., Hieret C. 2008, A\&A, 482, 179

Belloche A., Müller H. S. P., Menten K. M., Schilke P., Comito C., 2013, A\&A, 559, 47

Belloche A., Müller H. S. P., Garrod R. T., Menten K. M., 2016, A\&A, 587, 91

Belloche A., Garrod R. T., Müller H. S. P., Menten K. M., Medvedev I., Thomas J., Kisiel Z., 2019, A\&A, 628, 10

Bisschop S. E., Jorgensen J. K., van Dishoeck E. F., de Wachter E. B. M., 2007, A\&A, 465, 913

Bonfand M., Belloche A., Garrod R. T., Menten K. M., Stéphan G., Müller H. S. P., 2019, A\&A, 628, 27

Bossa J. B., Duvernay F., Theulé P., Borget F., D’Henkecourt L., Chinavassa T., 2009, A\&A, 506, 601

Brouillet N. et al., 2013, A\&A, 550, A46

Charnley S. B., Kress M. E., Tielens A. G. G. M., Millar T. J., 1995, ApJ, 448,232

Chengalur J. N., Kanekar N., 2003, A\&A, 403, 43

Chuang K.-J., Fedoseev G., Ioppolo S., van Dishoeck E. F., Linnartz H., 2016, MNRAS, 455, 1702

Chuang K.-J. et al., 2017, MNRAS, 467, 2552

Corby J. F. et al., 2015, MNRAS, 452, 3969

Enrique-Romero J., Rimola A., Ceccarelli C., Balucani N., 2016, MNRAS, 459, L6

Fedoseev G., Cuppen H. M., Ioppolo S., Lamberts T., Linnartz H., 2017, MNRAS, 448, 1288

Garrod R. T., Herbst E., 2006, A\&A, 457, 927
Garrod R. T., Weaver S. L.W., Herbst E., 2008, ApJ, 682, 283

Halfen D. T., Apponi A. J., Woolf N., Plot R., Ziurys L. M., 2006, A\&A, 639, 237

Halfen D. T., Ilyushin V. V., Ziurys L. M., 2013, ApJ, 767, 66

Halfen D. T., Woolf N., Ziurys L. M., 2017, ApJ, 845, 158

Herbst E., van Dishoeck E. F., 2009, ARA\&A, 47, 427

Hollis J. M., Lovas F. J., Jewell P. R., 2000, ApJ, 540, L107

Hollis J. M., Vogel S. N., Snyder L. E., Jewell P. R., Lovas F. J., 2001, ApJ, 554, L81

Hollis J. M., Lovas F. J., Jewell P. R., 2002, ApJ, 571, L59

Hollis J. M., Jewell P. R., Lovas F. J., Remijan A., 2004, ApJ, 613, L45

Jaber A. A., Ceccarelli C., Kahane C., Caux E., 2014, ApJ, 791, 29

Jones P. A. et al., 2008, MNRAS, 386, 117

Jones P. A., Burton M. G., Tothill N. F. H., Cunningham M. R., 2011, MNRAS, 411, 2293

Jørgensen J. K. et al., 2016, A\&A, 595, 117

Kaifu N., Morimoto M., Nagane K., Akabane K., Iguchi T., Takagi K., 1974, ApJ, 191, L135

Kauffmann J. et al., 2017, A\&A, 603, 89

Lee C.-W., Kim J.-K., Moon E.-S., Minh Y. C., Kang H., 2009, ApJ, 697, 428

Lefloch B., Ceccarelli C., Codella C., Favre C., Podio L., Vastel C., Viti S., Bachiller R., 2017, MNRAS, 469, L73

Li J. et al., 2017, ApJ, 849, 115

Martín-Pintado J., Vicente P. De, Rodríguez-Fernández N. J., Fuente A., 2001, ApJ, 548, L65

Meinert C. et al., 2016, Science, 352, 208

Menten K. M., 2011, in Pfalzner S., Kramer C., Straubmeier C., Heithausen A.eds, Proc. 4th Cologne-Bonn-Zermatt Symp. 241, The Dense Interstellar Medium in Galaxies. Springer, Berlin, p. 27

Miao Y., Snyder L. E., 1997, ApJ, 480, L67

Miao Y., Mehringer D. M., Kuan Y.-J., Snyder L. E., 1995, ApJ, 445, 59

Motiyenko R. A., Ilyushin V. V., Drouin B. J., Yu S., Margulès L., 2014, A\&A, 563, 137

Müller H. S. P., Schloder F., Stutzki J., Winnewisser G., 2005, J. Molecular Structure, 742, 215

Öberg K. I., Garrod R. T., van Dishoeck E. F., Linnartz H., 2009, A\&A, 504, 891

Pickett R. L. et al., 1998, J. Quant. Spectrosc. Radiative Transfer, 60, 883

Qin S. L., Wu Y. F., Huang M. H., Zhao G., Li D., Wang J. J., Chen S., 2010, ApJ, 711, 399

Redondo P. A., Largo A., Barrientos C., 2015, A\&A, 579, 125

Requena-Torres M. A. et al., 2006, A\&A, 455, 971

Requena-Torres M. A., Martín-Pintado J., Martín S., Morris M. R., 2008, ApJ, 672, 352

Rivilla V. M. et al., 2018, MNRAS, 475, L30

Rivilla V. M. et al., 2019, MNRAS, 483, L114

Rong J. L. et al., 2016, MNRAS, 455, 1428

Sánchez-Monge Á. et al., 2017, A\&A, 604, 6

Sandford S. A. et al., 1991, MNRAS, 371, 607

Sharma M. K., Sharma A. K., Sharma M., Chandra S., 2016, New Astron., 45,45

Skouteris D., Balucani N., Ceccarelli C., Vazart F., Puzzarini C., Barone V., Codella C., Lefloch B., 2018, ApJ, 854, 135

Snyder L. E., Kuan Y.-J., Miao Y., 1994, in Wilson T. L., Johnston K. J., eds, The Structure and Content of Molecular Clouds. Springer-Verlag, Berlin, p. 187

Snyder L. E. et al., 2005, ApJ, 619, 914

Taquet V. et al., 2015, ApJ, 804, 81

Widicus Weaver S. L. et al., 2017, ApJ, 232, 3

Woods P. M. et al., 2013, ApJ, 777, 90

Xue C., Remijan A. J., Burkhardt A. M., Herbst E., 2019, ApJ, 871, 112

Zuckerman B. et al., 1975, ApJ, 196, L99

This paper has been typeset from a $\mathrm{T}_{\mathrm{E}} \mathrm{X} / \mathrm{L} \mathrm{T} \mathrm{E} \mathrm{X}$ file prepared by the author. 